\title{
Agendas digitales en México: una necesidad para la inclusión social desde lo local
}

\author{
Digital Agendas in Mexico: a need for social inclusion from the grass roots
}

\author{
Rosa Amelia Domínguez ARTEAgA \\ El Colegio de Tamaulipas, Calzada General Luis Caballero No. 1540, Colonia Tamatán, Cd. Victoria, 87060 Tamaulipas (México), \\ ameliadguez@gmail.com
}

\begin{abstract}
Resumen
Se realiza un estado de la cuestión sobre las agendas digitales en México. Se analizó el portal oficial del gobierno y se realizó una búsqueda en Internet de las agendas digitales de cada estado para compararla con el registro del gobierno. Se analizan los planes estatales de desarrollo, así como también la información de fuentes primarias, como diarios digitales, portales de gobierno, portales de instituciones de educación superior y cámaras u organismos en Tecnologías de la información y las comunicaciones. Los resultados obtenidos muestran que la mayoría de las entidades no poseen agenda digital, y tampoco lo contemplan en los planes estatales de desarrollo. Se concluye que existe una necesidad de conformar agendas digitales en la mayoría de las entidades de la República Mexicana que impacten verdaderamente a la población
\end{abstract}

Palabras clave: Agendas digitales. Administración electrónica. Políticas de información. Tecnologías de la información y la comunicación. Sociedad de la información. Méjico.

\section{Introducción}

Las Tecnologías de la información y las comunicaciones (TICs) son herramientas de uso general y se utilizan para realizar un sin fin de actividades que impliquen el manejo de información. Se ha aceptado la idea de que estas herramientas tecnológicas agregan valor tanto a las actividades productivas, como a las sociales (CEPAL, 2010). Por su naturaleza transversal están presentes en todos los sectores de la sociedad, ofreciendo beneficios en temas como educación, gestión gubernamental, trabajo, empresa y hasta el del entretenimiento.

El papel del gobierno es determinante para el éxito en la implementación de programas que incluyan a las TICs. Sobre todo porque éstas poseen un gran potencial para la inclusión social, lo que lleva asociado un desafío: el reajuste de políticas públicas para la consecución de sociedades de la información (SI), pero centradas en la mejora de la calidad de vida de todas las personas. En este sentido, en los países interesados surgen las agendas digitales en la era global.

\begin{abstract}
The state of digital agendas in Mexico is presented and discussed. The official government portal was analyzed and, at the same time, each state entity was tracked in the Internet, and the subsequent findings were compared. This information was constrasted with the state development plans and complementary information from other primary sources, such as digital newspapers, government portals, etc. Results indicate that most entities do not have a digital agenda and they are not even considering one in their state development plans. Thus, there is a need to establish digital agendas in the majority of the entities of the Mexican Republic, so that digital policies achieve a positive impact on the population.
\end{abstract}

Keywords: Digital agendas. Information and communication technologies. E-Government. Information policies. Information society. Mexico.

México es un país emergente que todavía presenta un gran atraso en lo que a desarrollo digital se refiere. Para ello, la labor de los distintos niveles de gobierno (federal, estatal y municipal) es trascendente en la conformación de una estrategia digital local, para llevar al país a alcanzar una SI para todos los mexicanos.

A tenor de lo anterior, el presente trabajo tiene como objetivo principal conocer el estado de la cuestión de las agendas digitales de las 32 entidades federales de México de cara a la sociedad global. Para ello, se ofrece primeramente una descripción del desarrollo de políticas de información enfocadas en las TICs, y que se han venido a llamar políticas para la SI en el mundo, para después hablar de la situación en América Latina. Más adelante se presenta la situación vivida en México al interior del país, haciendo una búsqueda de las agendas digitales estatales y comparando la información obtenida con la registrada por la dependencia nacional encargada. Además, se profundiza en el papel que juegan estas iniciativas en el Plan Estatal de Desarrollo (PED). 
Fuentes primarias digitales, también se abordaron. Se termina con los resultados y conclusiones de dicho análisis.

\section{Políticas de información y las agendas digitales}

En el presente trabajo entendemos una política de información (PI) como aquella fundamentada sobre todo en las TICs, según la literatura encontrada (Cornella, 1998; Rowlands, 1996). La mayor motivación de un país para desarrollar una política de este tipo, es la necesidad de responder a los avances de las nuevas tecnologías y acompañar los cambios económicos, políticos y sociales que éstas están perpetrando en los diferentes países. Estas iniciativas describen la convergencia tecnológica, y cuya estrella principal es la Internet (Méndez, 2000, p. 6).

Caridad et al. (2001) afirman que existe una necesidad de políticas de información flexibles, que respondan a la emergencia y desarrollo de una tecnología, como lo es ahora la Internet. Y es que ésta se ha convertido en el indicador principal para medir a una sociedad de la información.

Lo más importante para estos autores es que toda política de información debe propiciar que las personas hagan válido su derecho a la información. Algunos términos utilizados para identificar este tipo de política son: Política de Información Federal, Políticas Nacionales de Información, Política para la Sociedad de la información o Agendas Digitales.

Para aportar una definición de estas iniciativas hemos elegido la que nos ofrece la CEPAL sobre Política para la SI (Perez y Hilbert, 2009, p. 308):

Se entiende que las políticas de sociedad de la información son las iniciativas que abordan ese concepto de manera integral, es decir que se orientan al acceso masivo a las TIC, a la capacitación de recursos humanos y a la generación de contenidos y aplicaciones electrónicas en los diversos sectores de la sociedad.

Podemos mencionar como ejemplo de este tipo de políticas la Política Nacional de EEUU The National Information Infrastructure: Agenda for action 1993 (Méndez, 2000, p. 4). En la Unión Europea, el Libro Blanco sobre Crecimiento, competitividad y empleo, y el informe Bangemann titulado Europa y la Sociedad Global de la Información. Recomendaciones al Consejo Europeo (Caridad, 1999).

En este sentido, la labor de los gobiernos ha sido determinante para embarcar a sus países hacia el progreso de una nación con el uso de las TICs. Así Manuel Castells reafirma que el Estado (como una organización política) puede ser una "fuerza dirigente de innovación tecnológica" (2005, p. 36).

\section{Agendas digitales en América Latina}

Quince años han trascurrido desde que los países de América Latina iniciaron sus intentos de una política pública de TICs. En ese periodo, los países interesados participaron en la Cumbre Mundial sobre la Sociedad de la Información (CMSI) en sus dos etapas, además de trabajar para que se diera la aprobación del Plan de Acción de la Sociedad de la Información en América Latina y el Caribe (eLAC). La evolución de estos planes se relata y sintiza gráficamente en CEPAL (2015).

Los estudios que analizan la situación de la SI en la región evalúan como significativo el avance en la aplicación masiva de la TICs en el desarrollo económico y social y ha habido logros en "la instalación de una infraestructura de información digital, la modernización del Estado, la digitalización de procesos económicos para aumentar la productividad y la calidad de la educación y la salud, y la gestión de los desastres naturales" (Peres y Hilbert, 2009, p. 388).

\begin{tabular}{|c|c|c|c|c|}
\hline País & $\begin{array}{l}\text { Lugar } \\
2012\end{array}$ & $\begin{array}{c}I D T \\
2012\end{array}$ & $\begin{array}{c}\text { Lugar } \\
2013\end{array}$ & $\begin{array}{c}I D T \\
2013\end{array}$ \\
\hline Dinamarca & 2 & 8,78 & 1 & 8,86 \\
\hline R. de Corea & 1 & 8,81 & 2 & 8,85 \\
\hline Suecia & 3 & 8,68 & 3 & 8,67 \\
\hline Islandia & 4 & 8,58 & 4 & 8,58 \\
\hline Reino Unido & 7 & 8,28 & 5 & 5,88 \\
\hline \multicolumn{5}{|c|}{ América Latina y el caribe } \\
\hline España & 29 & 7,14 & 28 & 7,38 \\
\hline Portugal & 39 & 6,57 & 43 & 6,67 \\
\hline Costa Rica & 55 & 5,64 & 55 & 5,92 \\
\hline Chile & 54 & 5,58 & 56 & 5,92 \\
\hline Argentina & 56 & 5,58 & 59 & 5,80 \\
\hline Brasil & 67 & 5,16 & 65 & 5,50 \\
\hline Colombia & 80 & 4,61 & 77 & 4,95 \\
\hline Venezuela & 78 & 4,68 & 80 & 4,81 \\
\hline Panamá & 77 & 4,69 & 82 & 4,75 \\
\hline Ecuador & 88 & 4,28 & 88 & 4,56 \\
\hline México & 94 & 4,07 & 95 & 4,29 \\
\hline
\end{tabular}

Tabla I. Índice de Desarrollo de las TICs 2014

Sin embargo, en varios países las iniciativas llevadas a cabo no han alcanzado el éxito esperado. Esta situación se ve reflejada en los bajos lugares que ocupa la región con respecto al desarrollo digital. La Unión Internacional de Telecomunicaciones (UIT) presentó el informe anual Medición de la sociedad de la información 2014, cuyos resultados muestran un gran atraso en 
comparación con los países desarrollados (UIT, 2014) (Tabla I).

Según la CEPAL (2010, p. 8), la situación que prevalece se debe a la falta de respaldo político e institucional. Además de lo anterior, el rezago en materia digital, es resultado de brechas de infraestructura y brechas de activos complementarios que todavía existen. Esta organización ha realizado un seguimiento para conocer los avances en el tema.

En CEPAL (2010, p. 103) se pueden visualizar las distintas agendas que han puesto en marcha los países de la región. Se discute que las mismas se han enfocado en reducir la brecha de acceso, pero que la de mayor aumento tiene que ver con los usos de las TICs (Rovira y Stumpo, 2013, p. 18). En consecuencia, existe un menor énfasis en las aplicaciones de TICs como facilitadoras del desarrollo económico.

Se considera que países como Colombia, Chile, Jamaica, México y el Uruguay poseen una mayor experiencia y continuidad en la formulación de políticas de este tipo. De los países de la región, Brasil ha sido pionero en la región al lanzar su Plan Nacional de Banda Ancha. Chile, Costa Rica, México, el Perú o la República Dominicana han realizado acciones en esa misma línea.

Actualmente la región se encuentra en una segunda generación de políticas para la sociedad de la información, aunque con pocas expectativas de alcanzar las metas propuestas para el 2015. Por tal motivo, en esa etapa del proceso de informatización de la sociedad, tiene relevancia absoluta el quehacer del gobierno en el establecimiento de iniciativas que guíen de forma eficaz estas acciones.

\section{Políticas de información en México y agendas digitales}

En México, el Plan Nacional de Desarrollo actual (PND, 2013-2018, p. 14), afirma que las TICs juegan un papel importante en el progreso nacional. $Y$ de las telecomunicaciones dicho documento (ibidem, p. 77), reafirma que "se han convertido en un insumo estratégico para competir en la economía moderna". Éste había sido el único documento rector que guiaba las políticas de información en nuestro país, aparte de los programas sectoriales implementados en años anteriores. Ahí mismo, se menciona que las entidades federativas harán lo propio en el ámbito de su competencia. Esto es importante debido a que las entidades estatales del país y sus municipios han jugado un papel muy importante en la instrumentación y uso de las TICs. Además, que su participación es esencial para extender los beneficios de las TICs y con ello disminuir la brecha digital interna que todavía acontece.

En 2013, gracias a la reforma en materia de telecomunicaciones y competencia económica, queda establecido que (DOF, 2013b, p. 1)

El Estado garantizará el derecho de acceso a las Tecnologías de la Información y Comunicación, así como a los servicios de radiodifusión y telecomunicaciones, incluido el de Banda Ancha e Internet.

En el mismo documento (ibidem, p. 9), se afirma que el Ejecutivo Federal tendrá a su cargo la política de inclusión digital universal.

Para realizar estas reformas, se tomó en cuenta que la densidad de la banda ancha en nuestro país figura entre las más bajas y lentas de los países de la OCDE. Asimismo, México se encuentra en los últimos lugares de penetración de banda ancha fija e inalámbrica (PND, 2013-2018, p. 55). Lo anterior es evidencia de los bajos niveles del país en desarrollo digital y es muestra de la necesidad de poner en práctica políticas públicas sobre TICs para aumentar los efectos de la digitalización para el progreso económico y social.

Por tal motivo, en el mismo año, se presenta la Estrategia Digital Nacional (EDN) (DOF, 2013a), cuyo objetivo es "lograr un México digital en el que la adopción y uso de las TICs maximicen su impacto económico social y político en beneficio de la calidad de vida de las personas" (EDN, 2013-2018, p. 9). Así, después de veinte años de que se anunciaran las primeras agendas digitales en los países desarrollados, por fin México tiene una política pública al respecto.

El atraso en el anuncio e implementación de una agenda nacional digital, va unido a lo que sucede con las entidades del país. En este sentido, para conocer la situación de las agendas digitales pero locales, existe muy poca literatura para abordar el tema. Por ejemplo, el estudio de Medina Romero (2012, p. 3) dirigido a la entidad de Michoacán. En su trabajo concluye que en esta entidad no existe una agenda digital "efectiva e integral que contribuya al desarrollo local". Para este autor tal situación se deriva de: una carencia de un marco jurídico-regulatorio que precise la agenda digital; inexistencia de un complejo institucional responsable de definir y ejecutar la agenda digital, que sea legal, legítimo y efectivo; insuficiente consideración de las estrategias digitales en el vigente PED para esa época.

Por el contrario, de lo que se ha escrito es de las estrategias de aprendizaje y gobierno electrónico, ya que en esa línea se han dirigido los esfuerzos en materia de política pública, en consonancia con lo que se vive en países de nuestra 
región. Lo que sí existen son varios estudios que analizan los portales de los gobiernos municipales como elementos para cercarse a los ciudadanos y gestionar mejor el gobierno (Luna, 2007, 2009).

Se afirma que ofrecer un ranking de los portales municipales, es relevante para las estrategias digitales estatales, debido a que estas herramientas constituyen una de las plataformas a través de las cuales este nivel de gobierno puede contribuir a la transformación gubernamental. Estos estudios de gobierno electrónico son los que han permitido visualizar y hablar de un estado de la cuestión en lo que al diseño de políticas de información locales se refiere (CIDGE, 2015).

En México, los esfuerzos relacionados con la implementación de las estrategias digitales en las entidades, así como las municipales, se realizan desde la Comisión Intersecretarial para el Desarrollo del Gobierno Electrónico (CIDGE). Esta instancia se conforma para consolidar el uso y aprovechamiento de las TICs en la Administración Pública Federal, promoviendo iniciativas alineadas a las nacionales, pero de acuerdo a las necesidades regionales.

Además, también se encarga de lo que sucede con las Agendas Digitales de las 32 entidades de la República Mexicana. Por lo tanto, la creación de una sociedad de la información local debe ser prioritaria, con el objetivo de definir las políticas públicas que permitan el acceso, uso y apropiación de las TICs por parte de la población (CIDGE, 2015).

Para tal fin, esta instancia pone al alcance de los municipios una guía que facilite la formulación de esta iniciativa. El documento ofrece pautas para determinar líneas de acción que permitan a estos niveles de gobierno, adoptar el uso de la tecnología "para generar, procesar y compartir información, que trastoque todos los ámbitos de la vida del país, como la pobreza, educación, salud, servicios gubernamentales, actividades económicas, y todo aquello que afecte la vida de los ciudadanos"(CIDGE, 2014).

\section{Metodología}

Para analizar el estado de la cuestión de lo que sucede al interior del país, el punto de partida es abordar el registro que existe en el CIDGE, debido a que es la única fuente oficial por parte del gobierno de la República Mexicana que informa al respecto. Se accedió a dicho sitio y se hizo la búsqueda en la primera quincena de julio del año 2015.

Para corroborar lo que el CIDGE había publicado, en el mismo periodo nos dimos a la tarea de hacer una búsqueda de las agendas digitales de cada entidad en un buscador general (Google). Las palabras clave para tal fin fueron 'agenda digital' y el nombre de la entidad. Se hizo una búsqueda por cada entidad de la República (31 y un Distrito Federal). La idea era saber si este organismo había publicado todas las existentes.

Por otro lado, analizamos los PED. La intención era conocer si en ellos estaba presente la idea de la creación de agendas digitales. Las palabras clave para tal fin fueron 'agenda digital', 'estrategia digital'. Es preciso mencionar que se puso atención en el año de inicio del PED y los años transcurridos hasta la fecha, y con ello se determina la importancia que se la ha dado a la política pública de este tipo.

Por ser un tema muy novedoso, se recurrió también a otras fuentes primarias, como diarios digitales, portales de gobierno, portales de instituciones de educación superior y cámaras u organismos en TICs. El objetivo fue conocer de primera mano los eventos ocurridos en las entidades, relacionados con el anuncio, diseño, presentación o implementación de estas agendas.

\section{Resultados}

En el momento de la búsqueda, el portal de la CIDGE registra que existen solo cuatro entidades con una agenda digital, representando el 13,3\% del total de las entidades del país. El portal de esta dependencia presenta una actualización al 9 de abril de 2015. Lamentablemente, no informa sobre la metodología utilizada para la obtención de estos datos.

La tabla II (en la página siguiente) nos muestra cuáles son las entidades que, según la CIDGE, poseen documentos donde se abordan temas sobre TICs o Gobierno Digital, así como aquellos que ya poseen una agenda digital, aunque no lo manifieste así el portal. Se distingue entre los planes estatales de desarrollo y los programas estatales especiales o estratégicos de difusión y fomento de la Ciencia, Tecnología e Innovación por entidad.

Al momento de buscar en la Red las agendas digitales por entidad, en primer lugar se encuentra que algunas de ellas ya han implementado agendas de este tipo. Coincidimos con el CIDGE, con una mínima diferencia. Por entidades, los hallazgos se dividen en los apartados siguientes: Agendas digitales no registradas en el CIDGE, agendas contempladas en los PED, agendas anunciadas en portales de gobierno y medios digitales. Estos apartados se detallan a continuación. 


\begin{tabular}{|c|c|c|c|}
\hline Entidad Federativa & $\begin{array}{c}\text { Plan } \\
\text { Estatal }\end{array}$ & $\begin{array}{c}\text { Programas } \\
\text { TIC }\end{array}$ & $\begin{array}{c}\text { Agendas } \\
\text { digitales }\end{array}$ \\
\hline Aguascalientes & • & & \\
\hline Baja California & • & & \\
\hline Baja California Sur & • & & \\
\hline Campeche & • & & \\
\hline Chiapas & $\cdot$ & & \\
\hline Chihuahua & $\cdot$ & • & \\
\hline Coahuila & • & & \\
\hline Colima & • & & \\
\hline \multicolumn{4}{|l|}{ Distrito Federal } \\
\hline Durango & • & & \\
\hline Estado de México & - & & \\
\hline Guanajuato & • & • & \\
\hline Guerrero & • & - & \\
\hline Hidalgo & • & & • \\
\hline Jalisco & - & & - \\
\hline Michoacán & • & & \\
\hline Morelos & $\cdot$ & & \\
\hline Nayarit & • & & \\
\hline Nuevo León & • & • & \\
\hline Oaxaca & • & & \\
\hline Puebla & - & & \\
\hline Querétaro & • & • & \\
\hline Quintana Roo & $\cdot$ & $\cdot$ & $\cdot$ \\
\hline San Luis Potosí & • & • & \\
\hline Sinaloa & $\cdot$ & • & \\
\hline Sonora & • & & \\
\hline Tabasco & $\cdot$ & & $\cdot$ \\
\hline Tamaulipas & $\cdot$ & & \\
\hline Tlaxcala & - & & \\
\hline Veracruz & • & & \\
\hline Yucatán & $\cdot$ & & \\
\hline Zacatecas & • & & \\
\hline
\end{tabular}

(PED, 2011-2016). Durango contempla una agenda digital, pero solo para educación. Tamaulipas no cuenta con agenda digital, pero el PED detalla acciones en diferentes áreas para crear una Sociedad del Conocimiento.

\subsection{Agendas digitales estatales anunciadas en portales de gobierno y medios digitales}

El portal de transparencia del Zacatecas, con información publicada al 11 de septiembre de 2013 y actualizada este año, habla de un departamento de agenda digital, así como la descripción de la misma. Desgraciadamente no se tiene más información al alcance, ni tampoco el documento que sustente lo publicado.

Algunos portales de gobierno de varias entidades informan de una Agenda digital, pero tampoco se encuentra documento al respecto. Tal es el caso de Colima, Distrito Federal, Guerrero, Puebla, San Luis Potosí y Veracruz.

El caso de Morelos llama la atención. Al hacer la búsqueda, el portal de gobierno anuncia que no se ha liberado la versión final del documento. Sólo ofrece portada e índice de la misma, pero no envía al interesado a otro sitio para completar la información.

La Cámara Nacional de la Industria Electrónica de Telecomunicaciones y Tecnologías de la Información (CANIETI) informa de una Agenda Digital para Yucatán. En Chihuahua un medio digital, con nota del año 2014, anuncia el lanzamiento de una agenda digital para esta entidad.

Tabla II. Agendas estales digitales registradas en el CIDGE

\subsection{Agendas digitales por entidad no registradas en el CIDGE}

En el primer caso se constata que el CIDGE no tiene registrada la agenda digital de la entidad de Baja California. Aunque la agenda no tiene fecha de realización, se considera que esta comisión debería actualizar el dato que ofrece.

\subsection{Entidades que contemplan en su PED la creación de agenda digital}

En Colima, la iniciativa digital se encuentra contemplada como el compromiso número ocho para la modernización del poder ejecutivo dentro del Plan Estatal de Desarrollo 2009-2015 (PED, 2009-2015, p. 148), y a dicho apartado se le ha denominado Agenda digital. También Zacatecas en su eje Zacatecas Moderno, establece como líneas estratégicas el fomento de una Sociedad y una Economía del Conocimiento e impulsar la incorporación de todos los sectores a las TICs

Un diario digital anuncia que en Chiapas se ha enviado ya una iniciativa de ley al congreso de la entidad, para la creación de la Agenda Digital.

\section{Conclusiones}

El atraso que han experimentado los países de la región tanto en la creación como en la concreción de políticas para la SI, se puede extrapolar hacia el interior de los mismos. Las entidades federativas, así como los municipios, también han vivido esta situación; han hecho frente a los cambios tecnológicos según se les iban presentando, sin dirección de la autoridad federal rectora. Lo anterior puede afectar la productividad y la competitividad a escala nacional, dando como resultado un retraso también en las entidades y en el ámbito municipal.

Se concluye que sólo cinco entidades de las que conforman la República Mexicana cuentan con una agenda digital. El resto, la mayoría de las entidades, no cuentan con una política de este tipo, ni contemplan su creación en los PED. Por el contrario, las entidades cuyo PED la contempla 
como una de sus metas, no la han llevado a cabo, mostrando poco interés en el tema. La mayoría de estos planes de gobierno son del 2009 a la fecha, tiempo suficiente para haberlos puesto en marcha en el presente.

La CIDGE registra las agendas digitales, aunque necesita actualizar los datos. Además también debe ofrecer información cualitativita de los planes y acciones en TICs de las entidades. La propuesta de investigación que surge a raíz de estos resultados es analizar también si las agendas digitales existentes son verdaderas políticas públicas que permitan incluir a México en la SI. Se recomienda un estudio de este tipo en un futuro.

Así, nos damos cuenta que en México existe una urgente necesidad de conformar agendas digitales integrales en las entidades, que miren por las necesidades locales. Esta situación limita el desarrollo económico, político, social y cultural de las regiones.

\section{Referencias}

Caridad Sebastián, Mercedes (1999). Planes de la Unión Europea para alcanzar el próximo milenio en Política del Conocimiento. // Caridad, Mercedes (Coord.). La Sociedad de la Información: Política, Tecnología e Industria de los Contenidos. Madrid: Centro de Estudios Ramón Areces, S. A., 1999, ISBN 84-8004-406-3.38-58.

Caridad Sebastián, Mercedes; Méndez Rodríguez, Eva Ma; Rodríguez Mateos, David (2001). La necesidad de políticas de información ante la nueva sociedad globalizada. El caso español. // Ciencia da informacao. ISSN 01001965. 29: 2,(maio/ago.2000) 22-36.

Castells, Manuel (2005). La era de la información, economía, sociedad y cultura. La sociedad red. ISBN 968-23-21689. vol.1.1-18

CEPAL [Comisión Económica para América Latina y el Caribe] (2010). Las TIC para el crecimiento y la igualdad: renovando las estrategias de la sociedad de la información. // Tercera Conferencia Ministerial sobre la Sociedad de la Información de América Latina y el Caribe Lima, 21 a 23 de noviembre de 2010. http://www.cepal.org/ddpe/publicaciones/xml/5/41725/LCG2464.pdf (2015-01-15)

CEPAL [Comisión Económica para América Latina y el Caribe] (2015). eLAC. Construyendo sociedades digitales inclusivas e innovadoras en América Latina y el Caribe. http://www.eclac.cl/cgibn/getprod.asp?xml=/elac 2015/noticias/pagi-

nas/2/44102/P44102.xml\&xsl=/elac2015/tpl/p18f.xsl\&bas e=/elac2015/tpl/top-bottom.xsl (2014-12-15).

CIDGE [Comisión Intersecretarial para el Desarrollo del Gobierno Electrónico] (2014). Guía para la formulación de la Estrategia Digital Estatal y Municipal. Versión 1.0. México, Secretaría de la Función Pública (SFP). http://cidge.gob.mx/wp-content/uploads/2015/06/Guiapara-la-Formulacion-de-la-EDEyM-V1.pdf (2015-07-11)

CIDGE [Comisión Intersecretarial para el Desarrollo del Gobierno Electrónico] (2015). México, Secretaría de la Función Pública (SFP). http://www.gob.mx/cidge/ (2015-0713)

Cornella, Alfons (1998). Políticas de información y rankings de países. // El Profesional de la Información. 1386-6710. 7:7-8 (julio-agosto 1996).
Dan entrada a iniciativa de ley de agenda digital para Chiapas. (13 de noviembre de 2012). // Diario sin embargo. http://www.sinembargo.mx/13-11-2012/429720 (201507-02).

México (2013a). Órgano del gobierno constitucional de los Estados Unidos Mexicanos. Decreto por el que se aprueba el Programa para un Gobierno Cercano y Moderno. Diario oficial de la Federación (DOF). Tomo DCCXIX No. 22 México, D.F., viernes 30 de agosto de $2013 . \quad$ http://pnd.gob.mx/wp-content/uploads/2013/11/Programa-para-un-Gobierno-Cercano-y-Moderno-2013-2018.pdf (2015-02-14)

México (2013b). Órgano del gobierno constitucional de los Estados Unidos Mexicanos. Decreto por el que se reforman y adicionan diversas disposiciones de los artículos 6o., 7o., 27, 28, 73, 78, 94 y 10 de la Constiticuión política de los Estados Unidos Mexicanos, en materia de Telecomunicaciones. // Diario oficial de la Federación (DOF) http://www.dof.gob.mx/nota_detalle.php?codigo $=5301941 \&$ fecha $=11 / 0 \overline{6} / 2013 \quad(2013-11-06) \quad(2015-$ 04-17)

México (2013). Gobierno de la República. Estrategia Digital Nacional. Noviembre de 2013. http://cdn.mexicodigital. gob.mx/EstrategiaDigital.pdf (2015-04-05)

México (2013). Gobierno de la República. Plan Nacional de Desarrollo 2013-2018. http://pnd.gob.mx/. (2015-04-07)

México. Distrito Federal (2013). Gaceta oficial. Décima Séptima época. 11 de septiembre de 2013. No. 1689, tomo II. http://www.consejeria.cdmx.gob.mx/portal_old/ uploads/gacetas/522fe67482e50.pdf (2015-07-02)

Baja California (2014). Gobierno del estado de Baja California. Agenda Digital Baja California. Tecnología para el Desarrollo y Competitividad. http://www.bajacalifornia. gob.mx/portal/doctos/ADBC.pdf (2015-07-12)

Colima (2009). Gobierno del estado de Colima Plan Estatal de Desarrollo 2009-2015. http://www.colima-estado.gob. $\mathrm{mx} /$ transparencia/archivos/plan_estatal_2009-2015.pdf (2015-07-07)

Chihuahua (2010). Gobierno del estado de Chihuahua. Plan estatal de desarrollo 2010-2016. http://transparencia. uach.mx/planeacion/plan_estatal_desarrollo_2010-2016. pdf (2015-07-03)

Durango (2011). Gobierno del estado de Durango. Plan Estatal de Desarrollo 2011-2016. http://www.ordenjuri dico.gob.mx/Documentos/Estatal/Durango/wo86894.pdf (2015-07-05)

Morelos (2012). Gubernatura del estado de Morelos. Agenda de Gobierno Digital. Una Nueva Visión, 2012 - 2018. Coordinación General de Gobierno Digital. 06/10/2012. http://agenda-egobierno.morelos.gob.mx/CGGD/Archivo s\%20\%20CGGD/agende\%20de\%20gobierno\%20digital/Agenda\%20de\%20Gobierno.pdf (2015-07-08).

Puebla. Congreso de Puebla. Presentan a Diputados la Agenda Digital del Gobierno del Estado. Comunicado 788. 25 de julio de 2012. http://www.congresopuebla.go b. $\mathrm{mx} /$ index.php?option $=$ com content\&view=article\&id=8762: presentan-a-diputados-la-agenda-digitaldel-gobiern

o-del-estado\&catid=100: noticias\&Itemid=128 (2015-0701).

San Luis Potosí. Gobierno del estado de San Luis Potosí. H. Ayuntamiento de San Luis Potosí. http://sanluis.gob.mx Inuevos-fraccionamientos-estaran-alineados-la-agendadigital/ (2015-07-07).

Tamaulipas (2011). Gobierno del estado de Tamaulipas. Plan Estatal de Desarrollo 2011-2016. Actualización octubre 2013. http://transparencia.tamaulipas.gob.mx/wp-content/uploads/2013/11/III-PED-TAMAULIPAS-Actualiz aci\%C3\%B3n-2013-2016.pdf (2015-07-08). 
Veracruz (2012). Gobierno del estado de Veracruz. http://www.veracruz.gob.mx/blog/2012/09/18/veracruzprimer-estado-con-una-agenda-digital-con-perspectivade-genero-secom/\#close (2015-07-10).

Zacatecas (2011). Gobierno del estado de Zacatecas. Plan Estatal de Desarrollo 2011-2016. http://www.ordenjuridi co.gob.mx/Documentos/Estatal/Zacatecas/wo86958.pdf (2015-07-08).

Zacatecas. Gobierno del estado de Zacatecas. Portal de transparencia de Zacatecas. http://transparencia.zacate cas.gob.mx/portal/?p=d\&inf=116577\#atencion $(2015-07$ 09).

ITU (2014). The ICT Development Index (IDI). https://www. itu.int/en/ITU-D/Statistics/Documents/publications/mis20 14/MIS_2014_Exec-sum-S.pdf (2015- 03- 15).

Luna-Reyes, Luis Felipe; Gil-García, Ramón; Rojas-Bandera, Jennifer (2007). An exploratory study of electronic government and state portals in Mexico. // M. Gascó- Hernández (Ed.). Latin America online: Cases, successes and pitfalls. Hershey, Pensilvania, Estados Unidos: Idea Group Inc. 2007. 116- 156

Luna-Reyes, Luis Felipe; Hernández García, Juan Manuel; Gil-García, Ramón (2009). Hacia un modelo de los determinantes de éxito de los portales de gobierno estatal en México. // Gestión y Política Pública. 18:2 (II semestre de 2009) 307-340.
Medina Romero, Miguel Ángel (2012). Agenda Digital, Marco Jurídico y Desarrollo para Michoacán: Lineamientos Estratégicos para Transitar a la Sociedad de la Información y el Conocimiento. // Revista Iberoamericana para la Investigación y el Desarrollo Educativo. 9, (jul-dic 2012) 114.

Méndez Rodríguez, Eva María (2000). Política del Tándem Clinton-Gore en Materia de Información: El liderazgo de los Estados Unidos./ / Mercedes Caridad Sebastián, (coord.). La sociedad de la Información: política, tecnología e industria de los contenidos. Madrid: Fundación Ramón Areces, 2000.

Naciones Unidas (2013). Objetivos de Desarrollo del Milenio: Informe de 2013. Nueva York. 63 p. http://www.un. org/es/millenniumgoals/pdf/mdg-report-2013-spanish.pdf (2014-08- 01). http://www.un.org/es/millenniumgoals/pdf/ mdg-report-2013-spanish.pdf (2014-08-01).

Perez, Wilson; Hilbert, Martin (2009). La sociedad de la información en América Latina y el Caribe. Desarrollo de las tecnologías y tecnologías para el desarrollo. CEPAL Santiago de Chile, febrero de 2009. http://repo sitorio.cepal.org/bitstream/handle/11362/2537/S0900902 _es.pdf?sequence=1 (2014-11-12).

Rovira, Sebastián; Stumpo, Giovanni (Compiladores) 2013). Entre mitos y realidades. TIC, políticas públicas y desarrollo productivo en América Latina. Naciones Unidas, CEPAL. http://www10.iadb.org/intal/intalcdi/PE/2013/107 67.pdf (2014- 10- 21).

Rowlands, I. (1996). Understanding information policy: concepts, frameworks and research tools. // Journals of Information Science. 22: 1. (February 1996)13-25.

Se llevó a cabo la presentación de la Agenda Digital Guerrero. (24 de septiembre de 2014). Diario de Guerrero. http://www.diariodeguerrero.com.mx/secciones/noticiasdel-dia/1847-se-llevo-a-cabo-la-presentacion-de-la-agen da-digital-guerrero\#sthash.7hMv5apy.dpuf (2015-07-09).

Torrero Ortega, Martha. CANIETI. Gobierno del Estado y Canieti elaboran Agenda Digital de Yucatán. 30 Oct 2012. http://www.canieti.org/comunicacion/noticias/vista/ 12-10-30/Gobierno_del_Estado_y_Canieti_elaboran_Ag enda_Digital_de_Yucat\%C3\%A프.aspx (2015-07-01).

Enviado: 2015-07-30. Segunda versión: 2016-06-14. Aceptado: 2016-09-15. 
